\title{
INSULIN RESISTANCE AND LIPID METABOLISM
}

Insulin resistance has been recognized as an important and independent risk factor for the development of type 2 diabetes and cardiovascular diseases ${ }^{1}$. This understanding has evolved over the last two or three decades, first in close relation with obesity and later on, also in the non-obese populations. Frequently, insulin resistance appears in the non diabetic population together with a cluster of risk factors including high blood pressure, chronic low grade inflammation and hyperlipidemia, which characterize the metabolic syndrome ${ }^{2}$. The most common and typical disorders of lipid metabolism in these cases are, indeed, the high triglyceride and low HDL levels, a combination that has long been described as responsible for the high risk of cardiovascular events. The physiological mechanisms for association of hypertriglyceridemia, low LDL levels and insulin resistance are becoming increasingly clear. Apart from the well discussed influence of insulin resistance on the activity of lipoprotein lipase, it seems that hyperlipidemia per se might be, conversely, a promoter of systemic inflammation, a cornerstone of the metabolic syndrome ${ }^{3}$.

From the clinical and epidemiological point of view, the recent increase of obesity worldwide calls for new therapeutical tools based on clinical facts and oriented by precise diagnostic methods. The article from Vasques and co-workers ${ }^{4}$ corroborates recent evidence, showing that high triglyceride levels and low HDL levels, or more specifically, the triglycerides/HDL ratio can be useful to predict insulin resistance calculated by means of the HOMA-IR index. These observations of great interest were obtained from a sample of the Brazilian population, which is very heterogeneous in terms of ethnicity, as emphasized by the authors. Moreover, the entire population studied was characterized by a normal or barely normal body composition as demonstrated by the $\mathrm{BMI}$ values, even in the highest quartile of the HOMA-IR index, above 1.29. This finding reinforces the hypothesis that this issue can no longer be considered as restricted to obesity.

These results suggest that monitoring triglyceride and HDLcholesterol levels is an interesting and probably useful tool to determine the concomitant occurrence of insulin resistance in the clinical setting. A pertinent question following this conclusion is whether the ratio will be able to indicate insulin resistance also in real life.. This can only be confirmed by additional populationbased studies. In the meantime it is worthwhile to focus attention on patients with high triglyceride and low HDL-cholesterol levels, even in the absence of obesity. They could be possible candidates for a detailed investigation of the carbohydrate metabolism, or more specifically, viewed as patients at high risk for the development of diabetes and cardiovascular complications.

Dimas IKEOKa Eva Krusinova

Fellow Scientists - Division of Endocrinology and Nuclear Medicine, Department of Internal Medicine, Medical University Graz. Austria

\section{Correspondence:}

Dimas Ikeoka

Stiftingtalstrasse 24

A-8010 -Graz

Austria

E-mail: dimas.ikeoka@healthsite.at

\section{References}

1.DeFronzo RA, Ferrannini E. Insulin resistance. A multifaceted syndrome responsible for NIDDM, obesity, hypertension, dyslipidemia, and atherosclerotic cardiovascular disease. Diabetes Care. 1991;14:173-94.

2.Day C. Metabolic syndrome, or What you will: definitions and epidemiology. Diabetes Vasc Dis Res. 2007;4:32-8.

3. Kopecky Jr J, Krusinova E, Klementova M, et al. Selected adipokines - plasma concentrations and adipose tissue expressions during 24-hour lipid infusion in healthy men. Physiol Res. 2009 [Epub ahead of print]. Available from: http:// www.biomed.cas.cz/physiolres/pdf/prepress/1464.pdf

4.Vasques ACJ. Indicadores do perfil lipídico plasmático relacionados à resistência à insulina. Rev.Assoc. Med.Bras.2009;55(3):342-6. 